\title{
BMJ Open POFA trial study protocol: a multicentre, double-blind, randomised, controlled clinical trial comparing opioid-free versus opioid anaesthesia on postoperative opioid-related adverse events after major or intermediate non- cardiac surgery
}

\author{
Helene Beloeil, ${ }^{1}$ Bruno Laviolle, ${ }^{2}$ Cedric Menard, ${ }^{3,4}$ Catherine Paugam-Burtz, ${ }^{5}$ \\ Matthias Garot, ${ }^{6}$ Karim Asehnoune, ${ }^{7}$ Vincent Minville, ${ }^{8}$ Philippe Cuvillon, ${ }^{9}$ \\ Sebastien Oger, ${ }^{10}$ Julien Nadaud, ${ }^{11}$ Sylvain Lecoeur, ${ }^{12}$ Gerald Chanques, ${ }^{13}$ \\ Emmanuel Futier, ${ }^{14}$ On behalf of the SFAR research network
}

To cite: Beloeil H, Laviolle B, Menard C, et al. POFA trial study protocol: a multicentre, doubleblind, randomised, controlled clinical trial comparing opioid-free versus opioid anaesthesia on postoperative opioid-related adverse events after major or intermediate non-cardiac surgery. BMJ Open 2018;8:e020873. doi:10.1136/ bmjopen-2017-020873

- Prepublication history for this paper is available online. To view these files, please visit the journal online (http://dx.doi. org/10.1136/bmjopen-2017020873).

Received 18 December 2017 Revised 23 April 2018 Accepted 21 May 2018

Check for updates

For numbered affiliations see end of article.

Correspondence to Professor Helene Beloeil; helene.beloeil@chu-rennes.fr

\section{ABSTRACT}

Introduction Reducing opioid consumption during and after surgery has been recommended for more than 10 years. Opioid-free anaesthesia (OFA) is a multimodal anaesthesia associating hypnotics, NMDA antagonists, local anaesthetics, anti-inflammatory drugs and $\alpha-2$ agonists. Proofs of the effect of OFA on reducing opioidrelated adverse effects after major or intermediate noncardiac surgery are still scarce. We hypothesised that the reduced opioid consumption allowed by OFA compared with standard of care will be associated with a reduction of postoperative opioid-related adverse events.

Methods/analysis The POFA trial is a prospective, randomised, parallel, single-blind, multicentre study of 400 patients undergoing elective intermediate or major non-cardiac surgery. Patients will be randomly allocated to receive either a standard anaesthesia protocol or an OFA. The primary outcome measure is the occurrence of a severe postoperative opioid-related adverse event within the first 48 hours after extubation defined as: postoperative hypoxaemia or postoperative ileus or postoperative cognitive dysfunction. In addition, each component of the primary outcome measure will be analysed separately. Data will be analysed on the intention-to-treat principle and a per-protocol basis.

Ethics and dissemination The POFA trial has been approved by an independent ethics committee for all study centres. Participant recruitment begins in November 2017. Results will be published in international peer-reviewed medical journals.

Trial registration number NCT03316339; Pre-results.

\section{INTRODUCTION}

Since the 1960s, intraoperative administration of opioids is considered a keystone of

\section{Strengths and limitations of this study}

The POFA trial is the first prospective, randomised, parallel, single-blind, multicentre study evaluating the effect of opioid-free anaesthesia (OFA) on severe postoperative opioid-related adverse events.

- The multicentre design, broad inclusion criteria, large sample size (400 patients) and follow-up will support external validity.

- The POFA trial is specifically studying OFA using dexmedetomidine. Other type of OFAs (ie, using clonidine) will not be tested.

anaesthesia as well as hypnotics and muscle relaxants. Synthetic opioids were introduced to achieve haemodynamic stability during anaesthesia. They allow an inhibition of the sympathetic system without cardiovascular collapse and histamine release. Since then, anaesthesia has changed from inhalation to multimodal anaesthesia with lower doses of hypnotic. In 2017, the intraoperative objectives of hypnosis, haemodynamic stability, immobility and anticipation of postoperative analgesia can be achieved without opioids. Moreover, opioid administration consequences are neither scarce nor benign for the patient. Perioperative opioids are associated with nausea and vomiting, ${ }^{1}$ sedation, ${ }^{2}$ ileus, ${ }^{3}$ confusion/delirium, ${ }^{4}$ respiratory depression, ${ }^{5}$ increased postoperative pain and morphine consumption, ${ }^{6}$ immunodepression, ${ }^{7}$ hyperalgesia and chronic postoperative 
pain. ${ }^{8}$ Among these complications, hypoxaemia, ileus and confusion/delirium are the most frequent. They are associated with a significant morbidity, can increase the length of stay and slow postoperative rehabilitation:

- Postoperative hypoxaemia is frequent in postanaesthesia care unit (PACU) ${ }^{10}$ It appears within minutes of tracheal extubation and the incidence is maximal 30 min after the arrival in PACU. The incidence of postoperative arterial hypoxaemia after abdominal surgery varies between $20 \%$ to $40 \%$ in the literature. ${ }^{11} 12$ The residual effect of anaesthetic drugs plays a major role in the genesis of early postoperative hypoxaemia, especially opioids. ${ }^{13}$ Postoperative opioid-induced respiratory depression is a cause of death and brain damage. Among 357 acute pain claims registered in the USA between 1990 and 2009, 92 were respiratory depression of which $77 \%$ resulted in severe brain damage or death. The vast majority $(88 \%)$ of respiratory depression occurred within 24 hours of surgery. ${ }^{5}$

- Postoperative ileus (POI) is a well-known consequence and complication of gastrointestinal, pelvic and some non-abdominal surgeries (ie, spine), resulting in significant morbidity and patient discomfort and dissatisfaction. More serious complications can include gastrointestinal perforation, nosocomial infections, malnourishment and muscular atrophy. These sequelae make POI one of the most important factors of prolonged hospitalisation following abdominal surgery. The development and consequences of POI following abdominal surgery is further complicated by the need for opioids to manage moderate to severe pain. Opioids are associated with bowel dysfunction, POI in non-abdominal procedures, and can exacerbate and prolong recovery from ileus following abdominal surgeries. A recent review revealed that, in selected surgeries, $10.3 \%$ of patients treated with opioids had ileus. ${ }^{3}$ Furthermore, higher doses of opioids were associated with higher incidence of POI.

- Delirium and postoperative cognitive dysfunction (POCD) are extremely common in geriatric surgical patients. After elective major joint replacement or other types of major surgery, about $5 \%$ to $15 \%$ of elders develop delirium and $25 \%$ to $40 \%$ and $12 \%$ to $15 \%$ develop, respectively, early or late POCD. Delirium and POCD are associated with prolonged length of stay, discharge to a place other than home and higher 1 year mortality. In addition, delirium is associated with an accelerated trajectory of cognitive decline to dementia. ${ }^{14}{ }^{15}$ Opioids are one of the risk factors of POCD. Patients receiving postoperative analgesia through a patient-controlled analgesia device that administers opioids intravenously were shown to be at significantly higher risk for the development of POCD. ${ }^{4}$ Moreover, a fast-track set-up with multimodal opioid sparing analgesia is associated with a lack of delirium after elective hip and knee arthroplasty in elderly patients. ${ }^{16}$
Efficacious multimodal analgesia and anaesthesia are the basis of successful fast-track surgery. These multidrug regimens aim at decreasing postoperative pain, intraoperative and postoperative opioid requirements, and subsequently, opioid-related adverse effects and hastening recovery. Opioid-free postoperative analgesia has therefore been recommended for more than 10 years. ${ }^{17}$ Based on the same principle of opioid sparing, opioid-free anaesthesia (OFA) is a multimodal anaesthesia associating hypnotics, N-methyl-D-aspartate (NMDA) antagonists, local anaesthetics, anti-inflammatory drugs and $\alpha-2$ agonists. Haemodynamic stability can be achieved without opioids during anaesthesia in 2017. The first studies on OFA focused on bariatric surgery where respiratory complications are frequent. OFA with dexmedetomidine (Dex) significantly attenuated postoperative pain and reduced opioid requirements without causing respiratory depression in obese patients. ${ }^{18} 19$ OFA was then proposed for awake neurosurgery ${ }^{20}$ and various minor ${ }^{21}$ or major surgeries. ${ }^{22}$ Two meta-analyses have concluded that intraoperative Dex reduced postoperative pain and opioid consumption. ${ }^{23}{ }^{24}$ One study showed a reduction of postoperative nausea and vomiting (PONV).$^{25}$ Adverse effects were hypotension and bradycardia. Proofs of the effect of OFA on reducing opioid-related adverse events after major or intermediate non-cardiac surgery are still scarce. We hypothesised that the reduced opioid used during and after surgery allowed by OFA compared with standard of care will be associated with a reduction of postoperative opioid-related adverse events.

\section{METHODS AND ANALYSIS \\ Trial design}

The POFA Study is an investigator-initiated, national, multicentre, randomised, single-blind, parallel-group clinical trial with concealed allocation of patients scheduled to undergo elective intermediate or major non-cardiac surgery 1:1 to receive either a standard anaesthesia protocol or an OFA. The trial will be conducted at 11 university and non-university centres. The study started in December 2017 and the recruiting period will be 24 months.

\section{Participant eligibility and consent}

Trial site investigators will identify consecutive eligible patients from the listed criteria. Eligible patients will receive written and oral information and will be included after investigators have obtained informed written consent.

\section{Inclusion criteria}

- Adult (18 years or older) patients admitted to the participating centre.

- Undergoing a scheduled major or intermediate non-cardiac surgery. ${ }^{26}$

- Benefiting from the health insurance system.

- Having signed an informed consent. 
Non-inclusion criteria

- Pregnant or breastfeeding women.

- Allergy to Dex or one of its excipients.

- Allergy to one of the drugs used for anaesthesia or one of their excipients.

- Urgent surgery.

- Intracranial surgery.

- Transplant surgery or transplanted patients.

- Surgery with planned regional anaesthesia.

- Outpatient surgery.

- Atrioventricular block, intraventricular or sinoatrial block.

- Adam-Stokes syndrome.

- Patients chronically treated with $\beta$-blockers and HR $<50$ bpm.

- Cardiac insufficiency with an left ventricular ejection fraction (LVEF) $<40 \%$.

- Epilepsy or seizures,

- Acute cerebral pathology.

- Obstructive sleep apnoea syndrome.

- Severe hepatic insufficiency (prothrombin ratio $<15 \%$ ).

- Adults legally protected (under judicial protection, guardianship or supervision), persons deprived of their liberty.

- Patients in whom the confusion assessment method for the intensive care unit (CAM-ICU) cannot be performed (ie, deaf patients).

- Uncontrolled hypotension.

\section{Allocation and blinding}

Patients will be randomised in two groups (control group and Dex group). In order to ensure group comparability, a plan of randomisation will be used. Randomisation will be done by investigators as close as possible to the surgery. Each patient will be given a unique patient number and a randomisation number (patient code) will be computer generated. It will be a block randomisation. Randomisation will be stratified on the centre and on the type of surgery: abdominal (digestive, urological, gynaecological) or non-abdominal. The primary evaluation criterion will be assessed blinded to the randomisation group. During the study period, patients and outcome assessors will be kept blind to the randomisation group. Nurses evaluating outcomes in PACU and in the ward will not participate in the anaesthesia and will not be aware of the randomisation group. They will be blind to the treatment. The anaesthesiologist and the nurse anaesthesiologist (care providers) will be the only ones not blinded. They will not participate in the assessment of the patients at any time.

At each participating centre, data will be collected and entered into the electronic web-based case report form (eCRF) by personnel trained for the trial or clinic (clinical research associate), blinded to the allocation group, under the supervision of the trial site investigators.

\section{Interventions}

All included patients will be allocated to one of the following two study groups:

- Control group: patients will receive a standardised anaesthesia protocol with remifentanil.

- Dex group: patients will receive a standard anaesthesia protocol with Dex.

Standardised intravenous induction of general anaesthesia will include

Propofol $1.5-2 \mathrm{mg} / \mathrm{kg}$, lidocaine $1.5 \mathrm{mg} / \mathrm{kg}$ (intravenous bolus), ketamine $0.5 \mathrm{mg} / \mathrm{kg}$ (intravenous bolus), cisatracurium $0.15 \mathrm{mg} / \mathrm{kg}$ (intravenous bolus before tracheal intubation), dexamethasone $8 \mathrm{mg}$ (intravenous bolus) and target-controlled infusion (TCI) of remifentanil (3-5 ng/mL) (Control group) or intravenous Dex 0.4-1.4 $\mu \mathrm{g} / \mathrm{kg}$ (Dex group).

\section{Standardised maintenance of general anaesthesia will include}

Desflurane, intravenous lidocaine $1.5 \mathrm{mg} / \mathrm{kg} / \mathrm{hour}$, intravenous ketamine $0.25 \mathrm{mg} / \mathrm{kg} /$ hour, intravenous cisatracurium as needed and TCI remifentanil $(2-5 \mathrm{ng} / \mathrm{mL}$ ) (Control group) or intravenous Dex $0.4-1.4 \mu \mathrm{g} / \mathrm{kg} / \mathrm{hour}$ intravenous (Dex group). At the end of the surgery, intravenous morphine $(0.05 \mathrm{mg} / \mathrm{kg})$ will be administered in the Control group. In both groups, intraoperative dose changes will be left to the anaesthesiologist in charge of the patient. Depth of anaesthesia (BIS, Covidien, France) and analgesia (ANI, Métrodoloris, France) will be monitored. The target of bispectral index (BIS) will range between 40 and 60 and ANI between 50 and 70 .

\section{Standardised postoperative protocol will include}

Extubation after verification of standard criteria

Spontaneous breathing with total expired volume $(\mathrm{TeV}) \geq 5-8 \mathrm{~mL} / \mathrm{kg}$, respiratory rate of $12-25 \mathrm{c} / \mathrm{min}$, absence of residual curarisation defined by $\mathrm{T} 4 / \mathrm{T} 1 \geq 90 \%$ (train-of-four), peripheral capillary oxygen saturation $\left(\mathrm{SpO}_{2)} \geq 95 \%\right.$ with $\mathrm{FiO}_{2} \leq 50 \%$, verbal and motor response to simple orders, temperature $\geq 36^{\circ} \mathrm{C}$. Extubation defines the $\mathrm{H} 0$ for assessment of the primary and secondary criteria events.

\section{Postoperative treatment}

Intravenous lidocaine $1.5 \mathrm{mg} / \mathrm{kg} /$ hour for 12 hours, paracetamol $(1 \mathrm{~g} / 6$ hours intravenous and then oral), nefopam (20 mg/6 hours intravenous and then oral), morphine titration in PACU according to routine standard of care, morphine intravenous PCA according to routine standard of care, ondansetron as a rescue medication in case of PONV. Patients will leave the PACU when Aldrete Score $>9$.

Decisions about all other aspects of patient care will be performed according to the expertise of the staff at each centre and to routine clinical practice to minimise interference with the trial intervention. Nevertheless, to avoid extremes of clinical practice, trial investigators will be strongly encouraged to apply intraoperative normothermia, multimodal postoperative analgesia (without 
regional anaesthesia) and prevention of PONV based on Apfel Score. Early postoperative resumption of fluids and solids will be encouraged.

\section{Outcome measures}

Primary outcome measure

The primary outcome measure will be the occurrence of a severe postoperative opioid-related adverse event within the first 48 hours after extubation defined as: postoperative hypoxaemia or POI or POCD. The onset of an opioid-related adverse event will be assessed blinded to the randomisation group.

Postoperative hypoxaemia is defined as therapeutic oxygen supplementation to maintain $\mathrm{SpO}_{2}>95 \%$ within the first 48 hours after extubation; the duration of oxygen treatment will also be recorded. ${ }^{27}$

POI is defined as an absence of flatus or stools within the first 48 hours after extubation.

POCD will be evaluated using CAM-ICU by a care provider (either anaesthesiologist or nurse). ${ }^{28} 29$ has been validated in multiple settings and is a widely used standardised method for identifying delirium with a high sensitivity of $94 \%$ (95\% CI $91 \%$ to $97 \%$ ), high specificity of $89 \%$ (95\% CI $85 \%$ to $94 \%$ ). The CAM algorithm consists of four items: (1) Acute onset or fluctuating course. (2) Inattention. (3) Disorganised thinking. (4) Altered level of consciousness. The diagnosis of delirium by CAM/ CAM-ICU requires a positive response to features 1 and 2 plus either 3 or 4 ; in these cases, the patients will be considered as presenting a POCD.

\section{Secondary outcomes measures}

- Each component of the primary outcome measure will be analysed separately.

- Number of episodes of postoperative pain (Numerical Rating Scale $\geq 3$ ) within 48 hours after extubation and at rest.

- Opioid consumption during the 48 hours following extubation.

- Time between the end of remifentanil or Dex administration and an Aldrete Score $>9$ (when applicable).

- Time between the end of remifentanil or Dex administration and extubation.

- Rate of unscheduled admission in intensive care unit.

- Number of PONV episodes during the 48 hours following extubation. Need for rescue antiemetic medication will be recorded.

- Hospital length of stay (max 28 days) defined as the number of days after extubation before first hospital discharge.

- Number of cardiac events during surgery (bradycardia defined as the number of episodes with atropine administration, hypotension defined as mean arterial pressure (MAP) $<65 \mathrm{~mm} \mathrm{Hg}$, hypertension defined as MAP $>90 \mathrm{~mm} \mathrm{Hg}$ ) and rescue medication.

\section{Ancillary immunological study}

An ancillary study will be performed in 42 patients enrolled in the coordinating centre to confirm or not preclinical data suggesting opioid-induced immunodepression $^{30}$ and therefore a potential interest of OFA in sparing immune function. Blood samples will be drawn at D0 (morning before the surgery) then D2 (morning 2 days later) and D7 (before surgery) then D2 and D7 (or the day the patient leaves the hospital if scheduled before D7). Three tubes of $5 \mathrm{~mL}$ of blood in agar-free lithium heparinate (green top) tubes will be drawn at each of the three time points. The tubes must be shipped within 2 hours at room temperature to spare unstable factors such as plasma cytokines or HLA-DR expression levels. Two immunophenotyping analyses will be done by flow cytometry on the blood samples: quantification of regulatory $\mathrm{T}$ cells and measure of their activation markers, quantification of the circulating myeloid subpopulations, notably the suppressive myeloid cells, and the measure of HLA-DR expression. Quantification of circulating immunosuppressive cells (by flow cytometry) will be performed by the 'Laboratoire de Suivi Immunologique des Thérapies Innovantes (SITI)' of Rennes University Hospital. After analysis, remaining blood samples will be destroyed according to SITI's procedures. Neither cells nor other blood-derived material will be conserved.

\section{Statistical analysis and sample size calculation}

Statistical analysis will be performed on all randomised and evaluated patients (intention-to-treat analysis). It will be performed with SAS v.9.4 software (SAS Institute, Cary, North Carolina, USA) in the Methodology/Biometrics department of the Inserm 1414 Clinical Investigation Centre of Rennes. A first overall descriptive analysis and analysis by group will be performed. This consists of separate estimates, numbers and percentages for qualitative variables, means, $\mathrm{SE}$, medians and interquartile intervals for quantitative variables. The normal feature of the distribution of quantitative variables is checked. Student's t-test or Mann-Whitney U test, if necessary, will be used to compare quantitative variables, and $\chi^{2}$ test or Fisher's exact test, if necessary, will be used to compare qualitative variables between two groups at inclusion. The primary end point (composite end point) will be compared between the two groups with the $\chi^{2}$ test. Two interim analyses after inclusion of a third and two-thirds of the patients, and one final analysis are planned. Stopping rules will use the $\alpha$ spending function with the O'BrienFleming boundary. The cumulative values of $\alpha$ for each analysis are: 0.00021 at the first analysis, 0.01202 at the second analysis and 0.04626 at the final analysis (nTerim, V.1.1, Statistical Solutions, Cork, Ireland). The trial will be stopped early if the significance of the $\chi^{2}$ test is below these $\alpha$ values. For the analysis of the other end points, the same strategy as for baseline comparisons will be used. In addition, censored end points (time to achieve an Aldrete Score $>9$ ) will be compared using the log-rank test. Continuous end points repeatedly measured during the study will be compared using a repeated measure two-way (time, group) analysis of variance. For all these analyses, adjustments can be made in case of heterogeneity at 
inclusion. Possible adverse events are coded according to the MedDRA classification and are the subject of a descriptive analysis. Except for the interim analyses, a P value $<0.05$ will be considered as statistically significant for all analyses.

\section{Missing values}

Missing data will not be replaced. Mixed models can be used in analysis of repeated data to avoid deleting subjects with any missing values.

\section{Sample size estimation}

One hundred and ninety-six patients per group will be needed to have $80 \%$ power, at a two-sided $\alpha$ level of 0.05 , to show a relative between-group difference of $40 \%$ in the composite primary outcome measure $(30 \%$ to $18 \%$ ), under the assumption of an overall incidence of $5 \%$ of POI (from $5 \%$ to $20.6 \%$ after major or intermediate non-abdominal and abdominal surgery, respectively), ${ }^{1011} 20 \%$ of postoperative hypoxaemia (from $20 \%$ to $40 \%$ depending on the surgical site) ${ }^{12} 13$ and $5 \%$ of postoperative delirium (from $3.6 \%$ to $30 \%$ after elective surgery and abdominal surgery, respectively), ${ }^{3}{ }^{514}$ thus $30 \%$ for the primary outcome measure. A total of 400 patients will be included to take into account non-evaluable patients. Patients undergoing a second surgery or dying within 48 hours without presenting the primary evaluation criteria will be considered as success in the analysis.

\section{Data registration}

Data will be entered into the eCRF by trial or clinical personnel under the supervision of the trial site investigators at each participating centre. From the eCRF the trial database will be established. Data collection will be monitored by trained research coordinators.

The following data will be registered:

\section{Baseline characteristics at randomisation}

Demographic data (age, height, weight, gender and body mass index); American Society of Anaesthesiologists physical status; type of surgery; significant comorbidities (cardiovascular, respiratory, neurological, psychiatric and/orabdominal disease, cancer, preoperative chemotherapy or radiotherapy).

\section{Intraoperative data}

Date of surgery, total doses of anaesthesia medications, doses of rescue medication (atropine, norepinephrine, epinephrine, ephedrine, antihypertensive medications), BIS and ANI values, ventilation data at the beginning and the end of the surgery (VtE, RR, post expiratory end pressure (Peep), inspired fraction of oxygen), duration of surgery and anaesthesia, and intraoperative complications (episode of bradycardia with atropine administration, hypotension (MAP $<65 \mathrm{~mm} \mathrm{Hg}$ ), hypertension (MAP $<90 \mathrm{~mm} \mathrm{Hg}$ ), shock (haemorrhagic, septic, cardiac, anaphylactic), clamping of a major vessel (aorta, vena cava), cardiac arrest, death, oxygen desaturation $\left(\mathrm{SpO}_{2}\right.$
$<90 \%$ for more than $5 \mathrm{~min}$ ), necessity to interrupt the procedure).

\section{Postoperative data}

Patients will be assessed once daily until the end of day 2 (48 hours).

The following data will be collected:

- Post OR care pathway (PACU, scheduled ICU admission, unscheduled ICU admission).

- Duration to obtain Aldrete Score $\geq 9$.

- Extubation time.

- Post PACU care pathway (surgical ward, scheduled ICU) admission, unscheduled ICU admission).

- Duration of stay in PACU.

- Episodes of oxygen desaturation defined by $\mathrm{SpPO}_{2}<95 \%$ with oxygen requirement during the first 48 hours after extubation.

- Time to first flatus and first stool.

- CAM-ICU daily during the first 48 hours after extubation.

- Episodes of PONV.

- Presence of a postoperative nasogastric tube. If yes, date and hour of withdrawal.

- Postoperative pain: episodes of numerical pain score $(\mathrm{NRS}) \geq 3$.

- Total morphine consumption during the first 24 hours and 48 hours after extubation.

- Length of stay (max 28 days).

- Death (until day 28).

\section{Patient withdrawal}

A participant who no longer agrees to participate in the clinical trial can withdraw the informed consent at any time without need of further explanation. Participants who will withdraw from the study will be followed up, according to routine clinical practice in each participating centre. In order to conduct intention-to-treat analyses with as little missing data as possible, the investigator may ask the participant which aspects of the trial he/she wishes to withdraw from (participation in the remaining follow-up assessments, use of already collected data). Whenever possible, the participant will be asked for permission to obtain data for the primary outcome measure. All randomised patients will be reported, and all data available with consent will be used in the analyses. If appropriate, missing data will be handled in accordance with multiple imputation procedures if missing data are greater than $5 \%$.

\section{Safety}

Every serious adverse event related to the studied treatment or not, expected or unexpected, must be reported within 24 hours by the investigator to the sponsor on a 'Serious adverse event' form on which will be indicated the date of occurrence, criterion of severity, intensity, relationship with the treatment (or the study) evaluated and the outcome. The period in which serious adverse events should be reported begins from the day of the written informed consent 
to the end of the follow-up ( 48 hours). Whenever a serious adverse event persists at the end of the study, the investigator must follow the patient until the event is considered resolved. The following events: postoperative hypoxaemia, POI and POCD will be recorded as primary evaluation criteria in the case report form. In order to avoid collection duplication, they will not be reported on the 'adverse event' page of the case report form. As planned in the study, they will be analysed at the time of interim analyses (two interim analyses after inclusion of a third and two-thirds of the patients) which will permit to show potential difference between the two groups during the study.

In addition, serious adverse events will be submitted to the data monitoring and safety committee (DMSC). The DMSC is independent of the trial investigators and will perform an ongoing review of safety parameters and overall study conduct. The DMSC comprises three independent clinicians (anaesthesiologists), a physician pharmacologist and a methodologist. The DMSC will be responsible for safeguarding the interests of trial participants, assessing the safety and efficacy of the interventions during the trial, and for monitoring the overall conduct of the clinical trial. To contribute to enhancing the integrity of the trial, the DMSC may also formulate recommendations relating to the recruitment/retention of participants, their management, improving adherence to protocol-specified regimens and retention of participants, and the procedures for data management and quality control. Recommendations for pausing or stopping the study will be made by the DMSC in case of serious adverse reactions and suspected unexpected serious adverse reactions.

All adverse events for which the investigator or the sponsor considers that a causal relationship with the investigational medicinal products can be reasonably considered, will be considered as suspected adverse reactions. If they are unexpected, they are qualified as being Suspected Unexpected SAR (SUSAR) and will be notified in a report by the sponsor to Eudravigilance (European pharmacovigilance database) and to the local regulatory agency within the regulatory time periods for reporting: immediate declaration if seriousness criteria is death or life-threatening condition, declaration within 15 days for other seriousness criteria.

\section{Data handling and retention}

Data will be handled according to the French law. All original records (including consent forms, reports of suspected unexpected serious adverse reactions and relevant correspondences) will be archived at trial sites for 15 years. The clean trial database file will be anonymised and maintained for 15 years.

\section{Patient and public involvement}

Patients and public were not involved in any of the phases of this study.

\section{ETHICS AND DISSEMINATION}

\section{Ethical and legislative approvals}

The POFA trial is registered in the European Clinical Trials Database (EudraCT 2017-001907-61) and at ClinicalTrials.gov with the trial identification number NCT03316339. Trial methods and results will be reported according to the Consolidated Standards of Reporting Trials (CONSORT) 2010 guidelines. $^{31}$

\section{Publication plan}

Scientific presentations and reports corresponding to the study will be written under the responsibility of the coordinating investigator of the study with the agreement of the principal investigators and the methodologist. The coauthors of the report and the publications will be the investigators and clinicians involved, on a pro rata basis of their contribution in the study, as well as the biostatistician and associated researchers. All trial sites will be acknowledged, and all investigators at these sites will appear with their names under 'the POFA investigators' in an appendix to the final manuscript. Rules on publication will follow international recommendations. ${ }^{32}$

\section{CONCLUSION}

The POFA trial is the first prospective, randomised, parallel, single-blind, multicentre study evaluating the effect of OFA on severe postoperative opioid-related adverse events. If POFA yields positive results, it would bring strong data to promote OFA, showing the benefits of OFA in terms of reduction of opioid-related adverse events, reduction of global morbidity, reduction of the economic burden associated with opioid-related adverse events, and reduction in length of stay would result in a collective benefit for future patients and could lead to significant changes in the standard of care in anaesthesia.

\section{Author affiliations}

${ }^{1}$ CHU Rennes, Pôle Anesthésie et Réanimation, Inserm, NuMeCan, CIC 1414 and Université de Rennes 1, Rennes, France

${ }^{2}$ Univ Rennes, CHU Rennes, Inserm, CIC 1414 (Centre d'investigation clinique de

Rennes, service de pharmacologie clinique), Rennes, France

${ }^{3} \mathrm{CHU}$ de Rennes, Pôle de Biologie, Rennes, France

${ }^{4}$ INSERM, UMR U1236, Université Rennes 1, EFS Bretagne, Rennes, France

${ }^{5}$ Département Anesthésie Réanimation, Assistance publique-Hôpitaux de Paris (AP-

HP), Hôpital Beaujon, Hôpitaux Universitaires Paris Nord Val de Seine, and Université Paris Diderot, Clichy, France

${ }^{6}$ Centre Hospitalier Universitaire (CHU) Lille, Pôle Anesthesie Réanimation, Lille, France

${ }^{7}$ Department of Anesthesiology and Critical Care Medicine, Centre hospitalier universitaire (CHU) de Nantes, Nantes, France

${ }^{8}$ Département d'Anesthésie et de Réanimation, Centre Hospitalier et Universitaire de Toulouse, Toulouse, France

${ }^{9}$ Service Anesthésie, Pôle Anesthésie Réanimation Douleur Urgence, Centre Hospitalier Universitaire Carémeau, Nimes, France

${ }^{10}$ Centre Hospitalier de Périgueux, Pôle Bloc Anesthésie Chirurgie, Service d'anesthésiologie, Perigueux, France

${ }^{11}$ Centre Hospitalier Metz Thionville, Pôle Anesthesie Réanimation, Metz, France

${ }^{12}$ Centre Hospitalier Yves le Foll, Pôle Anesthesie Réanimation, Saint-Brieuc, France

${ }^{13}$ Department of Anaesthesia and Critical Care Medicine, University of Montpellier

Saint Eloi Hospital, PhyMedExp, University of Montpellier, Montpellier, France 
${ }^{14}$ Département de Médecine Périopératoire, Université Clermont Auvergne, GreD, CNRS, Inserm U1103, Centre Hospitalier Universitaire (CHU) Clermont-Ferrand, Clermont-Ferrand, France

\section{Collaborators SFAR research network: all authors}

Contributors $\mathrm{HB}$ contributed to the conception and design of the research protocol. EF and GC provided critical input pertaining to the design of the trial interventions and procedures. CM designed the ancillary study. $\mathrm{HB}$ wrote the first draft of the protocol and this manuscript. $B L$ designed the statistical analysis plan. All authors CP-B, MG, KA, VM, PC, SO, JN, SL, GC, EF critically revised and modified the protocol and the article. They all approved the final version to be published.

Funding The POFA trial is supported by funding from French Ministry of Health (Programme Hospitalier de Recherché Clinique Nationnal (PHRC) 2016), CHU de Rennes, Direction de la recherche Clinique, 2 avenue Henri le Guilloux, 35033 Rennes Cedex, France. The sponsor had no role in the trial design, trial conduct, data handling, data analysis or writing and publication of the manuscript.

Competing interests None declared.

\section{Patient consent required}

Ethics approval The POFA trial was approved by the French National Safety and Drug Agency (Agence Nationale de Sécurité du Médicament (11 July 2017). By 4 September 2017, the study had been approved for all centers by a central ethics committee (Comité de Protection des Personnes lle-de-France II, Paris, France)

\section{Provenance and peer review Not commissioned; externally peer reviewed.}

Open access This is an open access article distributed in accordance with the Creative Commons Attribution Non Commercial (CC BY-NC 4.0) license, which permits others to distribute, remix, adapt, build upon this work non-commercially, and license their derivative works on different terms, provided the original work is properly cited and the use is non-commercial. See: http://creativecommons.org/ licenses/by-nc/4.0/

(C) Article author(s) (or their employer(s) unless otherwise stated in the text of the article) 2018. All rights reserved. No commercial use is permitted unless otherwise expressly granted.

\section{REFERENCES}

1. Apfel CC, Läärä E, Koivuranta $M$, et al. A simplified risk score for predicting postoperative nausea and vomiting: conclusions from cross-validations between two centers. Anesthesiology 1999;91:693-700.

2. Paqueron $X$, Lumbroso A, Mergoni $P$, et al. Is morphine-induced sedation synonymous with analgesia during intravenous morphine titration? Br J Anaesth 2002;89:697-701.

3. Gan TJ, Robinson SB, Oderda GM, et al. Impact of postsurgical opioid use and ileus on economic outcomes in gastrointestinal surgeries. Curr Med Res Opin 2015;31:677-86.

4. Wang Y, Sands LP, Vaurio L, et al. The effects of postoperative pain and its management on postoperative cognitive dysfunction. Am J Geriatr Psychiatry 2007;15:50-9.

5. Lee LA, Caplan RA, Stephens LS, et al. Postoperative opioid-induced respiratory depression: a closed claims analysis. Anesthesiology 2015;122:659-65.

6. Fletcher D, Martinez V. Opioid-induced hyperalgesia in patients after surgery: a systematic review and a meta-analysis. Br J Anaesth 2014;112:991-1004

7. Afsharimani $B$, Cabot $P$, Parat MO. Morphine and tumor growth and metastasis. Cancer Metastasis Rev 2011;30:225-38.

8. Joly V, Richebe P, Guignard B, et al. Remifentanil-induced postoperative hyperalgesia and its prevention with small-dose ketamine. Anesthesiology 2005;103:147-55.

9. van Gulik L, Ahlers SJ, van de Garde EM, et al. Remifentanil during cardiac surgery is associated with chronic thoracic pain $1 \mathrm{yr}$ after sternotomy. Br J Anaesth 2012;109:616-22.
10. Canet J, Gallart L, Gomar C, et al. Prediction of postoperative pulmonary complications in a population-based surgical cohort. Anesthesiology 2010;113:1338-50.

11. Xue FS, Li BW, Zhang GS, et al. The influence of surgical sites on early postoperative hypoxemia in adults undergoing elective surgery. Anesth Analg 1999;88:213-9.

12. Reeder MK, Goldman MD, Loh L, et al. Postoperative hypoxaemia after major abdominal vascular surgery. Br J Anaesth 1992;68:23-6.

13. Canet J, Ricos M, Vidal F. Early postoperative arterial oxygen desaturation. Determining factors and response to oxygen therapy. Anesth Analg 1989;69:207-12.

14. Crosby G, Culley DJ, Dexter F. Cognitive outcome of surgery: is there no place like home? Anesth Analg 2014;118:898-900.

15. Card E, Pandharipande $P$, Tomes $C$, et al. Emergence from general anaesthesia and evolution of delirium signs in the post-anaesthesia care unit. Br J Anaesth 2015;115:411-7.

16. Krenk L, Kehlet H, Bæk Hansen T, et al. Cognitive dysfunction after fast-track hip and knee replacement. Anesth Analg 2014;118:1034-40.

17. Kehlet H, Dahl JB. Anaesthesia, surgery, and challenges in postoperative recovery. Lancet 2003;362:1921-8.

18. Feld JM, Hoffman WE, Stechert MM, et al. Fentanyl or dexmedetomidine combined with desflurane for bariatric surgery. $J$ Clin Anesth 2006;18:24-8.

19. Hofer RE, Sprung J, Sarr MG, et al. Anesthesia for a patient with morbid obesity using dexmedetomidine without narcotics. Can $J$ Anaesth 2005;52:176-80.

20. Goettel N, Bharadwaj S, Venkatraghavan L, et al. Dexmedetomidine vs propofol-remifentanil conscious sedation for awake craniotomy: a prospective randomized controlled trial. $\mathrm{Br} J$ Anaesth 2016;116:811-21.

21. Bakan M, Umutoglu T, Topuz U, et al. [Opioid-free total intravenous anesthesia with propofol, dexmedetomidine and lidocaine infusions for laparoscopic cholecystectomy: a prospective, randomized, double-blinded study]. Rev Bras Anestesiol 2015;65:191-9.

22. Ohtani N, Yasui Y, Watanabe D, et al. Perioperative infusion of dexmedetomidine at a high dose reduces postoperative analgesic requirements: a randomized control trial. J Anesth 2011;25:872-8.

23. Blaudszun G, Lysakowski C, Elia N, et al. Effect of perioperative systemic $\alpha 2$ agonists on postoperative morphine consumption and pain intensity: systematic review and meta-analysis of randomized controlled trials. Anesthesiology 2012;116:1312-22.

24. Schnabel A, Meyer-Frießem CH, Reichl SU, et al. Is intraoperative dexmedetomidine a new option for postoperative pain treatment? A meta-analysis of randomized controlled trials. Pain 2013;154:1140-9.

25. Ziemann-Gimmel P, Goldfarb AA, Koppman J, et al. Opioid-free total intravenous anaesthesia reduces postoperative nausea and vomiting in bariatric surgery beyond triple prophylaxis. $\mathrm{Br} \mathrm{J}$ Anaesth 2014;112:906-11.

26. Kristensen SD, Knuuti J, Saraste A, et al. 2014 ESC/ESA Guidelines on non-cardiac surgery: cardiovascular assessment and management: The Joint Task Force on non-cardiac surgery: cardiovascular assessment and management of the European Society of Cardiology (ESC) and the European Society of Anaesthesiology (ESA). Eur Heart J 2014;35:2383-431.

27. Abbott TEF, Fowler AJ, Pelosi P, et al. A systematic review and consensus definitions for standardised end-points in perioperative medicine: pulmonary complications. Br J Anaesth 2018;120:1066-79.

28. Inouye SK, Leo-Summers L, Zhang Y, et al. A chart-based method for identification of delirium: validation compared with interviewer ratings using the confusion assessment method. J Am Geriatr Soc 2005;53:312-8

29. Inouye $\mathrm{SK}$, van Dyck $\mathrm{CH}$, Alessi CA, et al. Clarifying confusion: the confusion assessment method. A new method for detection of delirium. Ann Intern Med 1990;113:941-8.

30. Sacerdote P, Franchi S, Panerai AE. Non-analgesic effects of opioids: mechanisms and potential clinical relevance of opioid-induced immunodepression. Curr Pharm Des 2012;18:6034-42.

31. Schulz KF, Altman DG, Moher D, et al. CONSORT 2010 statement: updated guidelines for reporting parallel group randomised trials. BMJ 2010;340:c332.

32. International Committee of Medical Journal Editors. Uniform requirements for manuscripts submitted to biomedical journals. $N$ Engl J Med 1997;336:309-15. 